\section{Journal Watch}

\author{
Neil Wigglesworth' and Deborah Xuereb²
}

The Journal Watch feature is provided as a service to our readers. The intention is to highlight new research and other developments in infection prevention and control and related fields, published elsewhere. A brief description of each article and its main findings is given here; readers are encouraged to refer to the full published article for the details of the work. The authors and the editorial management group would welcome feedback and recommendations for articles to feature in this column; for comments and recommendations please contact neil.wigglesworth@ gmail.com or editor@ips.uk.net.

There hasn't been a Journal Watch section for a few issues; there's just so much fantastic content in Journal of Infection Prevention it's getting difficult to squeeze us in, but we're back and hope you'll find this selection of articles interesting. If there's a theme to this issue, it's probably revisiting subjects we've covered before as they continue to challenge us all as practitioners. The first article in this issue is a case in point as it refers to an annual scourge for many of us, the Norovirus outbreak.

Fraenkel CJ, Inghammar M, Sodelund-Strand A, et al. (2018) Risk factors for hospital norovirus outbreaks: Impact of vomiting, genotype and multioccupancy rooms. Journal of Hospital Infection; DOI: 10.1016/j. jhin.2018.01.011.

The issue of what leads to Norovirus outbreaks is one of perennial interest, especially when the season rolls around again, as it has at the time of writing. Regular readers of the Journal of Infection Prevention will recall the IPS' own work on this issue led by Dr Evonne Curran (Curran et al., 2015). This article by a Swedish group uses a case-control methodology, nested in an observational study to identify risk factors for outbreaks versus sporadic cases. The article starts with a potted revision of all things Norovirus which will be useful to new practitioners and as revision to all. The study was conducted between 2010 and 2012 (and has therefore taken an inordinate amount of time to report) and included all cases of Norovirus in eight hospitals with 192 inpatient wards. The study appears to be well designed and robust with clear definitions for cases, outbreaks and index cases; all of the isolates were genotyped and subjected to phylogenetic analysis. The settings sound similar to many found in the UK with single rooms to four bed bays. A

\author{
Journal of Infection Prevention \\ 2018, Vol. 19(2) 96-98 \\ (C) The Author(s) 2018 \\ Reprints and permissions: \\ sagepub.co.uk/journalsPermissions.nav \\ DOI: $10.1177 / 1757 \mid 77418760703$ \\ jip.sagepub.com
}

@SAGE range of possible risk factors were studied and included in a multivariable logistic regression with some sensitivity analyses to try and increase the rigour of index case identification. As with so many of the studies we read, the results are not surprising to a practitioner. Of almost 1000 cases of Norovirus identified, a large majority $(\sim 700)$ were part of either an outbreak or cluster (as defined), with the remaining 186 identified as sporadic. The key risk factors for an outbreak were: number of patients sharing a room with the index case; symptom onset after admission; vomiting; old age; co-morbidity; and genotype, with type GII.4 most associated with outbreaks. There was some interaction between genotype and vomiting and it may be that the ability to induce vomiting may be the reason for the importance of the GII.4 genotype. Much of this reinforces the message of IPC; early recognition and isolation remain key. There are some other interesting aspects to this study. They admit they couldn't measure compliance with IPC precautions, a common failing in studies, but conversely they benefited from the fact that all the hospitals share a common electronic health record and lab system - if only, I hear you cry! Finally, they are guilty of using the phrase 'borderline significance' when describing some of their results; don't listen to this, results are either statistically significant or they're not!

Two subjects that we have revisited more than once are contact precautions and isolation in general as well as the use of video surveillance and this study, again from Journal of Hospital Infection, combines the two.

Katanami Y, Hayakawa K, Shimazaki T et al. (2018) Adherence to contact precautions among different types of healthcare workers through video monitoring in a tertiary hospital. Journal of Hospital Infection; DOI: 10.1016/j.jhin.2018.01.001.

\footnotetext{
'Infection Prevention and Control, Guy's and St Thomas' NHS Foundation Trust, London, UK

2Infection Control Unit, Mater Dei Hospital, Malta
}

Corresponding author:

Neil Wigglesworth, Infection Prevention and Control, Guy's and St

Thomas' NHS Foundation Trust, Westminster Bridge Road, London SEI 7EH, UK.

Email: neil.wigglesworth@gstt.nhs.uk 
As discussed above, many observational and intervention studies in IPC often fail to measure the quality of compliance with IPC precautions and that can limit our faith in their findings. This study, which specifically addresses the issue of compliance, is interesting for many reasons. Video monitoring could easily be controversial and an ethical minefield, whether as research or in routine practice. The fact that didn't seem to be a major issue in this study may be cultural or it may be because the authors clearly addressed the ethical considerations in their methods. One of the strongest features of this study is that it wasn't restricted to a few clinical areas, but included most of the inpatient settings in the 781-bed hospital where it was undertaken. Only psychiatric, maternity and paediatric wards were excluded; perhaps because of the ethical issues associated with those settings, though the authors don't comment on this. The design of the study is simple enough; the cameras were placed to observe the donning of personal protective equipment (PPE) before entering a patient room (either a single room or cohort) and the video recordings were assessed for compliance with correct PPE. In addition, an e-learning system was adopted which included the choice and donning/doffing of PPE and staff had to pass a test at the end of the e-learning programme. Contact precautions were required for the range of infections that any IPC practitioner would recognise, such as multi-drug resistant organisms (MDRO) and Clostridium difficile, although interestingly they included glutamate dehydrogenase $(\mathrm{GDH})$-positive, toxin-negative $C$. difficile in their list. The study looked at the compliance by healthcare worker (HCW) type and the results have some surprises in them. Not surprisingly, overall compliance, despite full knowledge of the video surveillance, was poor-in the range of $34-50 \%$ for different aspect of PPE adherence. The results are broken down into a lot of detail by HCW and PPE type, but a few headlines are worth highlighting. The highest adherence was found in infectious disease (ID) doctors (not surprising as these were part of the IPC team) and cleaning staff. Nurses and nursing assistants were significantly less likely than other groups to don the appropriate PPE. Interestingly adherence was lower in the ICU setting than other settings for gowns and gowns and gloves combined. It's also worthy of note and not surprising that, generally, adherence was higher when patients had diarrhoea - self-protection, again, a possible motivator. Finally, and least surprising of all, the use of the e-learning system appeared to make no difference to compliance; we know that knowledge doesn't equate to behaviour.

Although the focus of the next publication is staff uniform contamination, it makes an interesting finding on the role of contact precautions in the acquisition of microorganisms.

Thom KA, Escobar D, Boutin MA et al. (2017) Frequent contamination of nursing scrubs is associated with specific care activities. American Journal of Infection Control; DOI: 10.1016/j.ajic.2017.11.016.

I was drawn to this publication mainly as it addresses a topic that often raises interest and questions from members of staff: what is the role of uniforms in cross-transmission? At times, this study seems to look for the obvious but it's methodologically sound and worth a read. Over a period of eight months, this single-centre observational study from the USA, analysed a large dataset of 720 samples from 90 HCWs who had direct patient contact in an intensive care setting. Samples were taken during the last $4 \mathrm{~h}$ of the HCWs' shifts, from the front of the scrub top and down the front of both thighs using double-tipped swabs and agar plates. Identification and susceptibility testing were carried out for the bacteria that grew and total colony counts were recorded. The staff had interacted with an average of 5.5 patients during their shift. The majority had washed their scrubs before wearing. Patient activity before the sampling was recorded and the majority had cared for patients under contact precautions, who had a wound or were on a ventilator.

It's not surprising that $30 \%$ of the scrubs were contaminated with pathogenic bacteria, the most common being S. aureus (16\%) and gram-negative bacteria (16\%). Six percent of the total samples grew a multidrug-resistant organism. HCWs who gave a bath or cared for a patient with a wound were more likely to have pathogenic bacteria cultured from their scrubs, thus linking contamination to types of patient care activities. One could, however, question which PPE were worn during these care activities and whether these needed to be reviewed. The study also found that $\mathrm{HCW}$ caring for patients under contact precautions are more likely to be have their uniforms contaminated with pathogenic bacteria rather than other bacteria. The most interesting finding of this study was the significantly reduced colony counts of bacteria on uniforms of $\mathrm{HCW}$ caring for patients under contact precautions, signalling that the use of PPE potentially reduces the overall bio burden of contamination. This finding supports the value of contact precautions in decreasing acquisition of microorganisms by HCW. The authors call for further exploration of this finding especially at a time when contact precautions are being questioned. While reading this study, I was curious to know what were the bacteria most frequently cultured in patients on the unit as this could have influenced the type of bacteria cultured from uniforms. Also, at the time of study, there was no ICU-wide bathing of patients with chlorhexidine so it would be interesting to see if there were any changes after chlorhexidine baths were implemented. This study contributes to the discussion on the role of uniforms as a reservoir of pathogenic organisms and potential source for transmission. It still remains, however, methodologically difficult to prove that uniform contamination is linked to cross-transmission or whether it leads to infection in additional patients. 
The next publication builds on the topic of the effectiveness of PPE within a context of highly infectious diseases such as Ebola.

Hall S, Poller B, Bailey C et al. (2018) Use of UV fluorescence based simulation in evaluation of personal protective equipment worn for first assessment and care of a patient with suspected high consequence infectious disease. Journal of Hospital Infection; DOI: 10.1016/j. jhin.2018.01.002.

Most of us remember the hullabaloo during the Ebola outbreak around PPE recommendations for preparedness training; at one point it seemed the PPE combinations were changing every day. This publication tested the level of protection offered by PPE ensembles recommended by several UK hospitals when caring for patients with suspected high consequence infectious diseases (HCID). I found this study quite intriguing as it challenges the basic assumption in infection prevention that PPE combinations protect the wearer from contamination. Using a UV-florescence based simulator to visualise contamination of PPE and/or body area not only enabled contamination to be visualised, but also the source of the contamination to be identified. Qualitative feedback from HCWs with regards to PPE practicalities was also collected thus increasing the robustness of the conclusions. This study identified challenges with all the PPE ensembles tested. It concludes that models with lots of individual PPE elements and many donning and doffing steps require a higher level of concentration and offer more opportunities for mistakes and confusion. To reduce the number of workers at risk of contamination, it was considered preferable that the buddy should ideally be an observer and instructor but not physically assist in doffing. Analysis of the results showed breaches were related either to protocol failure or complications in PPE doffing, and provided conclusive evidence of the need for improvements. Indeed, the findings will be used as evidence to justify the proposal of a unified PPE ensemble for HCWs across the UK. The publication manuscript gives a lot of detail on how the tests were carried out and the findings and makes it a worthwhile read for those involved in training staff in HCID preparedness. The study recommends more rigorous simulation studies, as well as standardised doffing procedures and training advice. We anticipate a further publication and HSE guidance on a unified PPE ensemble based on the findings of this study.

The last paper in this selection is a short and simple one, but one that illustrates a simple and almost universal truth; to use the language of social media: \#vaccineswork.

Akhtar T, Cargill J, Gerrard C et al. (2018) The detection of rotavirus in paediatric oncology patients with diarrhoea: the impact of rotavirus vaccine. Journal of Hospital Infection; DOI: 10.1016/j.jhin.2017.12.022.

Once again, no apologies for going over old ground, it bears repeating. We reported on the impact of the UK Rotavirus vaccination programme in an earlier Journal Watch (Wigglesworth and Xuereb, 2016). This paper reinforces those findings and highlights their effect on a highly vulnerable population; children being investigated and treated for malignancies. The authors remind us that diarrhoea is a significant complication in their population and that until very recently, Rotavirus was the commonest cause of diarrhoea in children. Once again, there's a short primer on both Rotavirus and the vaccines that are available to prevent it, useful to newer practitioners and interesting revision for all of us. This was a simple retrospect review of 819 Rotavirus samples and 46 positive results. The results show a year-on-year reduction from $10 \%$ of samples positive in 2010 (before the vaccine introduction) to almost none in the last two years. In fact, in 2015 and 2016 they sent 122 samples and only had one single positive result. The authors conclude, and it's hard to argue with them, that there is very little if any utility in continuing to test for Rotavirus. The authors also conducted a survey of other paediatric oncology centres with regard to testing protocols and found a very mixed pattern of testing and requests, the detail of which can be found in the original article. We ended the previous comment on this issue (in 2016) with these words, and I don't think we need to change them: '...demonstrate once again the power of vaccination and immunisation. We can keep hoping for the day when scientists vaccinate us all out of a job...'

Once again there's a familiarity to the subjects we've covered on this issue; in part that's been deliberate, as some areas of IPC and wider public health practice, such as vaccination, deserve to be repeatedly highlighted because they continue to challenge us.

\section{Declaration of conflicting interests}

The author(s) declared no potential conflicts of interest with respect to the research, authorship, and/or publication of this article.

\section{Funding}

The author(s) received no financial support for the research, authorship, and/or publication of this article.

\section{References}

Curran ET, Wilson J, Haig CE, McCowan C, Leanord A and Loveday H. (2015) The Where is Norovirus Control Lost (WINCL) Study: an enhanced surveillance project to identify norovirus index cases in care settings in the UK and Ireland. Journal of Infection Prevention 17: $8-14$.

Wigglesworth N and Xuereb D. (2016) Journal Watch. Journal of Infection Prevention 17: 90-92. 